\title{
SeqURE - a new copy-capture based method for sequencing of unknown Retroposition events
}

\author{
Alexander Y. Komkov ${ }^{1,2^{*}}$ D, Shamil Z. Urazbakhtin', Maria V. Saliutina', Ekaterina A. Komech', Yuri A. Shelygin ${ }^{3}$, \\ Gaiaz A. Nugmanov', Vitaliy P. Shubin ${ }^{3}$, Anastasia O. Smirnova', Mikhail Y. Bobrov ${ }^{4}$, Alexey S. Tsukanov ${ }^{3}$, \\ Anastasia V. Snezhkina ${ }^{5}$, Anna V. Kudryavtseva ${ }^{5}$, Yuri B. Lebedev and Ilgar Z. Mamedov ${ }^{1,2,4,6^{*}}$
}

\begin{abstract}
Background: Retroelements (RES) occupy a significant part of all eukaryotic genomes including humans. The majority of retroelements in the human genome are inactive and unable to retrotranspose. Dozens of active copies are repressed in most normal tissues by various cellular mechanisms. These copies can become active in normal germline and brain tissues or in cancer, leading to new retroposition events. The consequences of such events and their role in normal cell functioning and carcinogenesis are not yet fully understood. If new insertions occur in a small portion of cells they can be found only with the use of specific methods based on RE enrichment and highthroughput sequencing. The downside of the high sensitivity of such methods is the presence of various artifacts imitating real insertions, which in many cases cannot be validated due to lack of the initial template DNA. For this reason, adequate assessment of rare $(<1 \%)$ subclonal cancer specific RE insertions is complicated.

Results: Here we describe a new copy-capture technique which we implemented in a method called SeqURE for Sequencing Unknown of Retroposition Events that allows for efficient and reliable identification of new genomic RE insertions. The method is based on the capture of copies of target molecules (copy-capture), selective amplification and sequencing of genomic regions adjacent to active RE insertions from both sides. Importantly, the template genomic DNA remains intact and can be used for validation experiments. In addition, we applied a novel system for testing method sensitivity and precisely showed the ability of the developed method to reliably detect insertions present in 1 out of 100 cells and a substantial portion of insertions present in 1 out of 1000 cells. Using advantages of the method we showed the absence of somatic Alu insertions in colorectal cancer samples bearing tumor-specific L1HS insertions.
\end{abstract}

Conclusions: This study presents the first description and implementation of the copy-capture technique and provides the first methodological basis for the quantitative assessment of RE insertions present in a small portion of cells.

Keywords: Retroelements, Human genome, Insertional polymorphism, Copy capture, High-throughput sequencing

\footnotetext{
* Correspondence: alexandrkomkov@yandex.ru; imamedov@mx.ibch.ru

'Shemyakin-Ovchinnikov Institute of Bioorganic Chemistry, Moscow, Russia

Full list of author information is available at the end of the article
}

(c) The Author(s). 2020 Open Access This article is licensed under a Creative Commons Attribution 4.0 International License, which permits use, sharing, adaptation, distribution and reproduction in any medium or format, as long as you give appropriate credit to the original author(s) and the source, provide a link to the Creative Commons licence, and indicate if changes were made. The images or other third party material in this article are included in the article's Creative Commons licence, unless indicated otherwise in a credit line to the material. If material is not included in the article's Creative Commons licence and your intended use is not permitted by statutory regulation or exceeds the permitted use, you will need to obtain permission directly from the copyright holder. To view a copy of this licence, visit http://creativecommons.org/licenses/by/4.0/ The Creative Commons Public Domain Dedication waiver (http://creativecommons.org/publicdomain/zero/1.0/) applies to the data made available in this article, unless otherwise stated in a credit line to the data. 


\section{Background}

Retroelements (REs) in the human genome comprise more than 1.5 million copies that arise as a result of a process called retrotransposition. Three major classes of REs are LTR retroposons, LINEs, and SINEs that include the primate-specific Alu family. Only a tiny portion of REs is still capable of retrotransposition including more than one hundred of autonomous LINE-1 (L1) copies [1] and non-autonomous Alu and SVA that recruit the L1 enzymes for retrotransposition. The activity of these copies results in new L1, SVA and Alu insertions found in the genome of newborns [2, 3] and also leads to somatic insertions in cancer [4-7] or normal tissues [8-11]. These insertions can be neutral, can cause genetic disorders, or could promote the development of cancer. The role of RE insertions in the generation of neuronal plasticity during adult neurogenesis is also considered [1214]. In the past decade, several methods for screening for new RE insertions have been developed. Most modern methods are based on high-throughput sequencing and can be divided into targeted and non-targeted. Nontargeted methods for new RE insertions discovery includes analysis of whole genome (WGS) or exome sequencing data with various software tools $[2,5,15,16]$. This approach is quite reliable if a new insertion is supported by many different sequencing reads which means that WGS data have a high sequencing coverage. Insertions present in a low percentage of cells in a sample are detected with less confidence and must be supported by independent methods such as locus-specific PCR combined with Sanger sequencing. In most cases with standard WGS coverage non targeted methods allow to find insertions present in $25 \%$ of cells or more [16]. Targeted methods of $\mathrm{RE}$ insertion detection include enrichment in $\mathrm{RE}$ sequences or their unique genomic flanking regions (flanks) prior to sequencing [14, 17-22]. The enrichment methods are based on either hybridization to RE specific oligos or selective amplification of RE flanks. These methods are more sensitive in respect of the catching somatic insertion events and obviously are more cost-effective compared to WGS. However, all the existing enrichment techniques can generate various artificial sequences (chimeras) that mimic true RE insertions and lead to many false-positive results [23, 24]. Thus, independent confirmation of insertions by locusspecific PCR starting from initial genomic DNA samples with subsequent Sanger sequencing and identification of Target Site Duplication (TSD - the sign of true retrotransposition event) became a gold standard in somatic RE studies [23]. In the case of insertions present in the genome of very few cells in the sample, this confirmation can be problematic [13] since genomic fragments containing a new RE insertion could be absent in another aliquot of the same DNA sample. In many cases, the initial sample is limited that restricts the number of locus-specific PCR reactions necessary to prove the identified insertions. Here we present a new method called SeqURE (Sequencing of Unknown Retroposition Events) for the detection of young $R E$ insertions in the human genome that overcome these limitations. The method is designed to amplify both $5^{\prime}$ and 3 ' flanking sequences from the same DNA sample and to identify TSD in the sequenced library. We demonstrate the power of our approach on the retroelements of Alu subfamilies. We also developed the first original protocol for measuring the RE identification methods' sensitivity. Using this protocol for Alu insertions we evaluated the sensitivity of our method of detecting Alu insertions at the level of $1 \mathrm{RE}$ insertion per 1000 cells. This protocol could be easily implemented for the testing of other RE identification methods.

\section{Results}

\section{Principle of the method}

The method is based on consecutive selective amplification of both $5^{\prime}$ and $3^{\prime}$ flanking regions adjacent to the copies of retroelements belonging to young RE subfamily of interest (Fig. 1). For detection of Alu (AluYa5 + AluYa8, hereafter AluYa5 or AluYb8 + AluYb9, hereafter AluYb8) genomic DNA is fragmented by a mixture of endonucleases FspBI and Csp6I which do not cut inside the Alu element. This combination of enzymes cleaves 92\% of the known AluYa5 and AluYb8 5'-flanks into fragments $25-800 \mathrm{bp}$ long (Fig. 2a). On the one hand, such length is suitable for the efficient cluster generation and sequencing on Illumina, and on the other hand, in most cases it is enough for RE insertions mapping to the genome. Additionally, $87 \%$ of known AluYa5 and AluYb8 have 3' flanks of the same length and $81 \%$ have both flanks in the range of detection. Moreover, 98\% of AluYa5 and AluYb8 insertions can be identified using either one or the other flank. Thus, despite using nonrandom fragmentation the power of the developed approach is comparable with the methods based on random DNA fragmentation such as ME-Scan [17]. We prefer DNA digestion by site specific restrictases to random sheering. Despite this approach leads to the loss of insertions having very short or very long flank (i.e. genomic distance between the insertion point and the nearest restriction site) it has some advantages over random fragmentation for identification of rare events. First, using a restrictase with the known restriction site prevents fragmentation of the element itself which is critical for both flanks amplification (see below). Second, using of restriction enzymes allows for the identification of chimeric sequences resulting from genomic fragments ligation that can mimic real insertion events. 


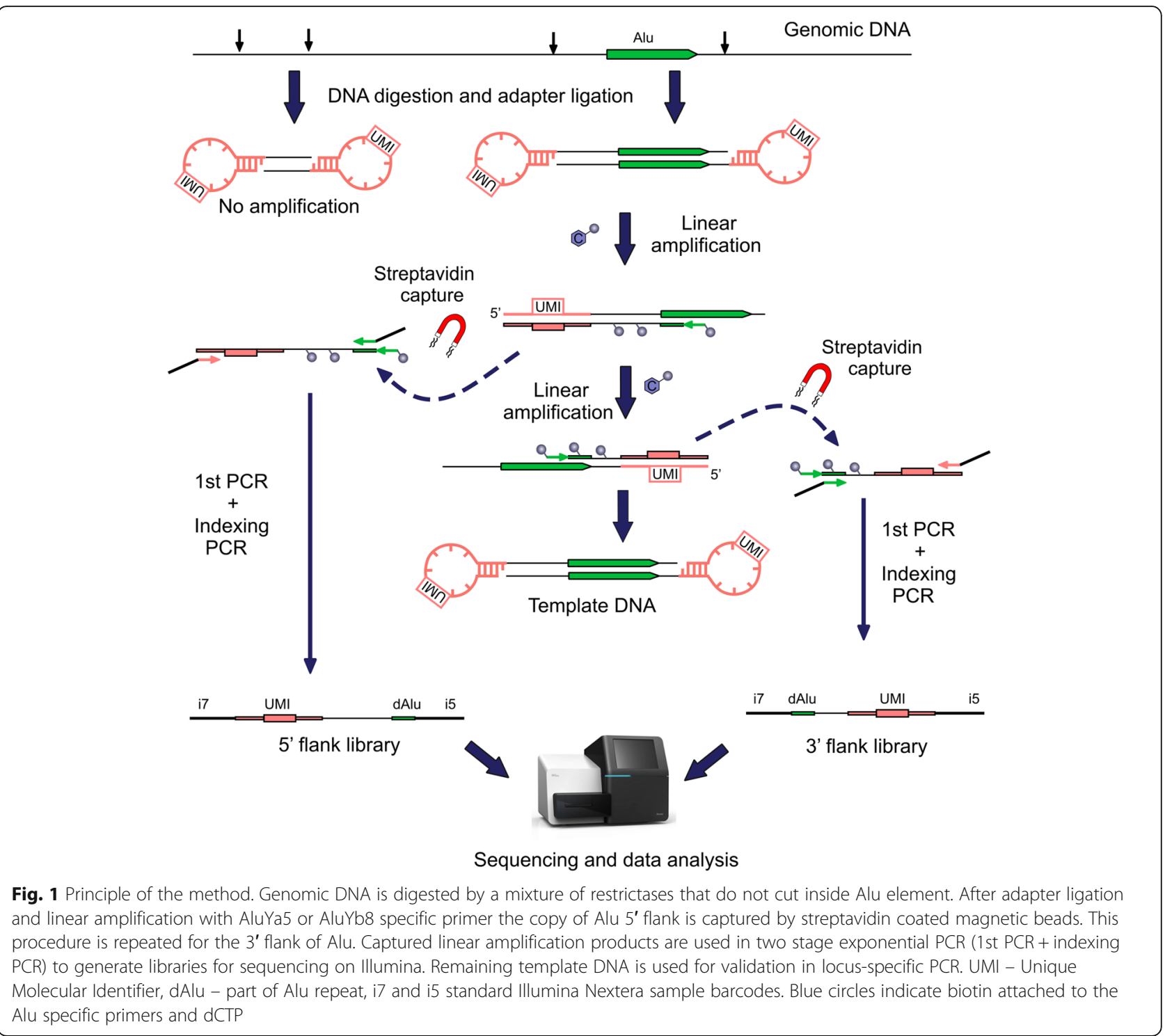

At the next step fragmented DNA is ligated to stemloop DNA adapters containing Unique Molecular Identifiers (UMI) [25]. These random $10 \mathrm{nt}$ oligonucleotides are used to label each molecule containing an insertion with a unique "molecular barcode", to remove PCR duplicates and to evaluate the number of cells bearing each insertion. The stem-loop adapter structure was designed to increase the ligation efficiency and thus maximize the chance to ligate the adapter to both ends of each DNA molecule. This oligo contains sequence identical to the primer used in the first PCR reaction that prevents amplification of molecules without target Alu sequence similar to "vectorett PCR" approach. At the next stage remaining adapters are inactivated by the ligation to anti-SL oligo. This procedure prevents residual adapters from priming amplification reaction at the next step and thus artificial increasing of UMI counts.
Next, the biotinylated primer specific to AluYa5 (or AluYb8) fragment containing 3 diagnostic mutations for Ya5 (or specific $7 \mathrm{nt}$ fragment for Yb8) is used for linear amplification of retroelement $5^{\prime}$ flanks. The biotinylated primer along with the biotinylated $\mathrm{dCTP}$ is used to introduce biotin into generated ssDNA molecules. After linear amplification the obtained product is denatured, the biotinylated $5^{\prime}$ flank copies are captured and removed from the reaction with the use of streptavidincoated magnetic beads. Importantly, the restricted and adaptor-ligated genomic DNA template remains intact. This DNA is used for the second linear amplification with the primer complementary to the same region characteristic for AluYa5 (or AluYb8) but in the opposite direction (towards the 3' flank of the insertion). This product is also captured by streptavidin-coated magnetic beads. The remaining initial template DNA is used to 


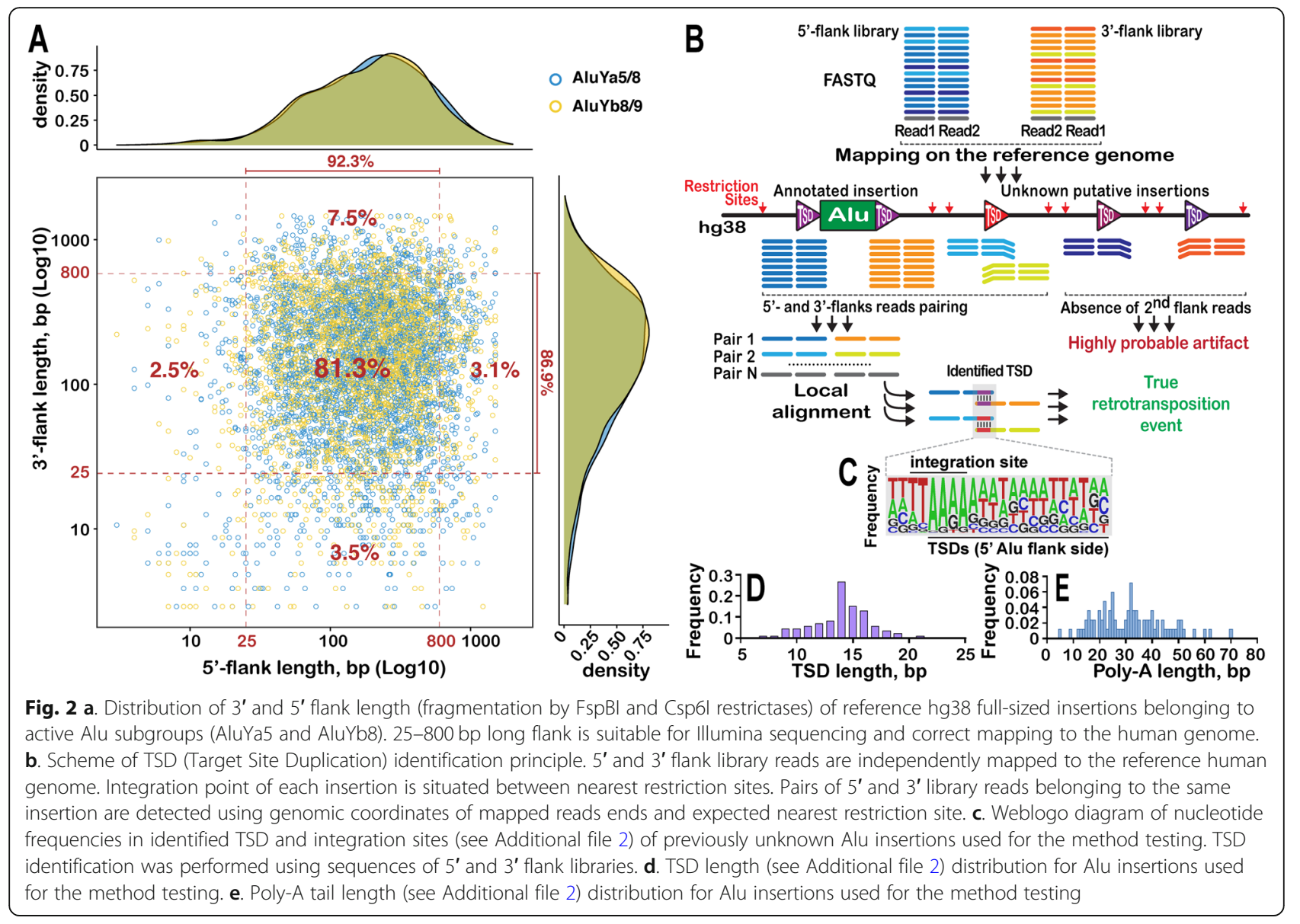

confirm insertions by locus-specific PCR. Captured linear DNA is amplified in the subsequent PCR to generate libraries of AluYa5 (AluYb8) flanks (separately for 5' and 3' flank). The second PCR is used for sample barcoding and generates libraries ready for sequencing on Illumina. The resulting fragments consist of a short part of the Alu repeat, adjacent genomic flank ( $5^{\prime}$ or $\left.3^{\prime}\right)$, a part of the adapter with UMI, and standard parts necessary for Illumina sequencing including sample barcodes. 3' flanking library also includes the polyA tail of the Alu.

After sequencing, we use a custom computational pipeline [24] to map all sequenced insertions flanks to the human genome. Coordinates of insertions in compared samples (e.g. normal vs tumor tissue, motherfather-children, etc.) are matched to identify samplespecific insertions. Importantly, our pipeline is able to filter out most artificial chimeric sequences that mimic real RE insertions. At the last stage, sample-specific insertions from both $5^{\prime}$ and $3^{\prime}$ flanks libraries are matched. Matched sequences adjacent to the Alu insertion are used to identify TSD. The number of UMI for each insertion is also counted to evaluate the percentage of cells in a sample bearing each insertion.

\section{Development of the protocol for testing method sensitivity}

To evaluate method sensitivity, we used polymorphic Alu insertions. Using our method we first identified coordinates of AluYa5 insertions in the genomes of four healthy individuals (D1, D2, D3 and D4). Then we compared these coordinates and identified in total 137 AluYa5 insertions that are absent in the genome of individual D1 and present in the genome of one of the individuals D2, D3, or D4 (see Table 1 for numbers and Additional file 1 for the detailed description of each detected insertion). Twenty five out of these 137 insertions are reference insertions present in the hg38 human genome. Eighty one AluYa5 are previously known non-reference insertions found in dbRIP (http:// dbrip.brocku.ca/) or 1000 genomes project databases [26]. The remaining 31 insertions are unknown non-reference polymorphic or germline insertions discovered in this study

Table 1 Number of Alu insertions present in genomes of individuals D2-D4 and absent in the genome of individual D1

\begin{tabular}{llll}
\hline & D2 (1\%) & D 3 (0.3\%) & D 4 (0.1\%) \\
\hline AluYa5 & 44 & 45 & 48 \\
Cell counts & $1.11-1.42 \%$ & $0.31-0.33 \%$ & $0.05-0.14 \%$ \\
\hline
\end{tabular}


for the first time. Next, we made a mixture of $\mathrm{T}$ cells containing 50,000 cells of individual D1, 500 cells of individual D2 (1\%), 150 cells of individual D3 (0.3\%), and 50 cells of individual D4 (0.1\%) using FACS sorting. Cells of each individual were stained by antibodies with different fluorescent dyes (see Methods). Mixing was done in five replicates. Three of them where re-run on FACS to evaluate the accuracy of sorting (see Table 1, cell counts and Methods). DNA from the other two replicates was extracted and 1/10 aliquots (the equivalent of 5000 mixed cells) were used to prepare libraries of Alu Ya5 5' and 3' flanks with SeqURE method.

After sequencing (see Table 2 for sequencing read numbers) and data processing (as described previously in [24]) we searched for $5^{\prime}$ flanks of Alu insertions characteristic for genomes of individuals D2-D4 (from Table 1 ) in the corresponding datasets. As a result, we were able to identify 44 out of 44 insertions present in $1 \%$ (individual D2) of cells, 42-43 out of 45 insertions present in $0.3 \%$ (individual D3) of cells and 34-39 of 50 insertions present in $0.1 \%$ (individual D4) of cells (See Table 2 ). The insertion was considered "found" if it has at least 2 sequencing reads corresponding to its flank in the dataset. The 5' flanking fragment length (genomic distance between insertion coordinate and the closest restriction site) in all 137 tested Alu insertions was between 30 and $529 \mathrm{bp}$.

Next, we searched for the same insertions in the 3' Alu flanks library. Obviously, a portion of these insertions have their restriction sites located too far from the integration point and their flanks are lost during amplification and Illumina sequencing. Thus, we first analyzed the distribution of restriction sites in the 3' flanks of Alu insertions identified in the $5^{\prime}$ flank library. We found that 36 out of 44 Alu for individual D2 (1\%), 37 out of 45 Alu for individual D3 (0.3\%) and 36 out of 48 Alu for individual D4 $(0.1 \%)$ have nearest 3 ' restriction site in the range of $30-529 \mathrm{bp}$ (same as for the 5'flank). Next, we searched the 3'flank library for the insertions that are present in both $5^{\prime}$ and $3^{\prime}$ flank libraries. As a result, we were able to find $32-34$ out of 36 insertions present in
1\% (D2) of cells, 26-29 out of 37 insertions present in $0.3 \%$ (D3) of cells and 16-18 out of 36 insertions present in $0.1 \%$ (D4) cells (See Table 2). Next, we compared sequences which are directly adjacent to each insertion from $5^{\prime}$ and $3^{\prime}$ side and were able to identify TSD for $87 \%$ of AluYa5 found in both $5^{\prime}$ and $3^{\prime}$ libraries (Fig. 2b, Additional file 2). For the remaining insertions the quality of the sequencing read following polyA track was insufficient to reliably identify TSD.

To evaluate the sequencing depth that is required for confident identification of Alu insertions we performed downsampling experiments on the $5^{\prime}$ flanks Alu datasets. For this purpose, we randomly selected $15,10,5,2$, $0.5,0.2$, and 0.1 millions of raw sequencing reads from 5 ' Alu flank datasets obtained from two mixed cells replicates and searched for the individual-specific insertions (see Fig. 3). The insertion was considered "found" if it had at least 2 sequencing reads corresponding to its flank in the dataset.

For $1 \%$ of cells, we found $100 \%$ insertions in both replicates with a sequencing depth of 10 million reads. The vast majority (39-40 out of 44 in a single replicate and 36 in both replicates) of insertions present in $1 \%$ of the cells in a sample can still be found with sequencing depth of 2 million reads. As expected, for the cells with 10 times lower concentration $(0.1 \%)$ even 15 million of reads is not enough to catch $100 \%$ of the insertions, however, 28 out of 48 (approximately 60\%) are repeatedly found in both replicates. Around $20 \%$ of insertions are still repeatedly detectable with sequencing depth of 2 million in both replicates (Fig. 3).

Reproducibility of the method was evaluated by the comparison of two independent replicates (independent cell mixtures). We plotted normalized UMI count in replicate 1 versus UMI count in replicate 2 for each of the identified Alu insertion from individuals D1-D4 (Fig. 4). Overall correlation coefficient between two replicates was $r^{2}=0.97$. For the insertions present in minor percentage of cells it was $r^{2}=0.75, r^{2}=0.43$, and $r^{2}=$ 0.17 for $1,0.3$ and $0.1 \%$, respectively.

Table 2 Number of individual-specific insertions found in 5' and 3' Alu flank libraries

\begin{tabular}{|c|c|c|c|}
\hline & Individual 2 & Individual 3 & Individual 4 \\
\hline \multicolumn{4}{|l|}{ Found in $5^{\prime}$ flank library } \\
\hline Replicate 1 (15,372,014 reads) & $44(44)^{a}$ & $43(45)$ & $39(50)$ \\
\hline Replicate 2 (20,116,093 reads) & $44(44)$ & $42(45)$ & $34(50)$ \\
\hline Both Replicates & $44(44)$ & $41(45)$ & $29(50)$ \\
\hline \multicolumn{4}{|c|}{ Found in both $5^{\prime}$ and $3^{\prime}$ flank libraries } \\
\hline Replicate 1 (18,644,933 reads) & $34(36)$ & $26(37)$ & $18(36)$ \\
\hline Replicate 2 (19,315,147 reads) & $32(36)$ & $29(37)$ & $16(36)$ \\
\hline Both Replicates & $32(36)$ & $23(37)$ & $11(36)$ \\
\hline
\end{tabular}

\footnotetext{
a - Number of expected insertions are given in parentheses
} 


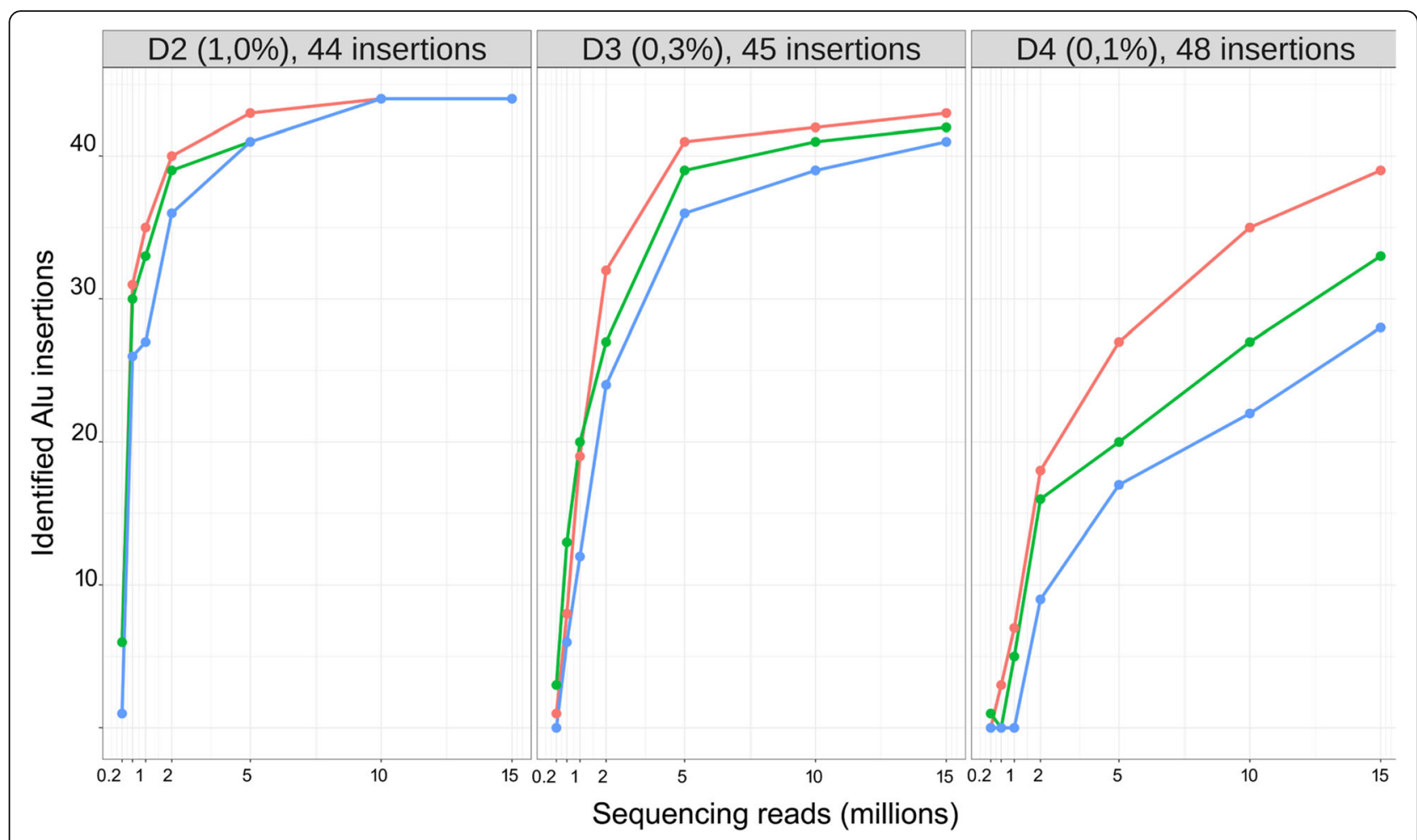

Fig. 3 Results of downsampling experiments on the 5' Alu flank datasets. Number of raw sequencing reads used in each sampling experiment is indicated on X axis. Red line - the number of individual specific Alu insertions found in replicate 1, green line - in replicate 2, and blue line in both replicates simultaneously

Initial DNA template (restricted genomic DNA with ligated adaptors) that left after two rounds (5' and $3^{\prime}$ flank) of linear amplification and capture was used as a template for locus-specific PCR. We selected 10 nonreference $\mathrm{Alu}$ insertions which have a distance between $5^{\prime}$ and 3' flanks restriction sites in the range 400-600 bp. Next, we removed short (100-300 bp) template DNA molecules from the mixture by magnetic beads. This procedure depletes DNA fragments that do not contain Alu insertions (empty allele) originating from the genome of individual D1. After that, we performed locus-specific PCR with the primers corresponding to unique flanking regions of each insertion. We observed PCR product of expected length for 4 out of 4 insertions present in the genome of D2 (1\% of cells), for 3 of 4 insertions present in the genome of D3 ( $0.3 \%$ of cells) and for 1 of 2 insertions present in the genome of D4 $(0.1 \%$ of cells). Obtained PCR products were sequenced by Sanger method which in all cases allowed to confirm the insertions by the alternative method and were able to identify TSD indicating true Alu insertions.

Identification of tumor-specific Alu insertions in colorectal cancer samples

Using our method, we performed a search for cancerspecific Alu Ya5 and AluYb8 insertions in 6 paired (tumor/normal) colorectal cancer samples. Libraries of 5' and 3' flanks were prepared using SeqURE method and sequenced. For each 5 ' flank library we obtained a minimum of 2,173,258 reads and the difference between tumor and normal samples in each pair was less than 2fold. After data processing and artifact filtration, we compared lists of Alu insertion coordinates in paired normal and tumor samples. Only the insertions with 2 or more different UMI present in the tumor sample and absent in corresponding paired normal sample were considered tumor-specific candidates. We identified 10 potential tumor-specific Alu insertions in the $5^{\prime}$ flank libraries of 5 out of 6 tumor samples (see Table 3). Using the same approach as for the method sensitivity assay, we searched for corresponding sequences in 3' flank libraries. For 7 out of 10 candidates identified in the $5^{\prime}$ flank libraries, we found no corresponding sequences in the 3' flank library. These candidates are most probably artifacts that are not filtered by our pipeline. For the other 3 candidates, we found corresponding $3^{\prime}$ sequences in both tumor and normal libraries. These insertions could represent non-reference polymorphic or germline insertions present in both normal and tumor cells but detected only in the tumor 5 ' flank library due to insufficient sequencing depth. For 8 candidate insertions, we designed primers and performed locus-specific 


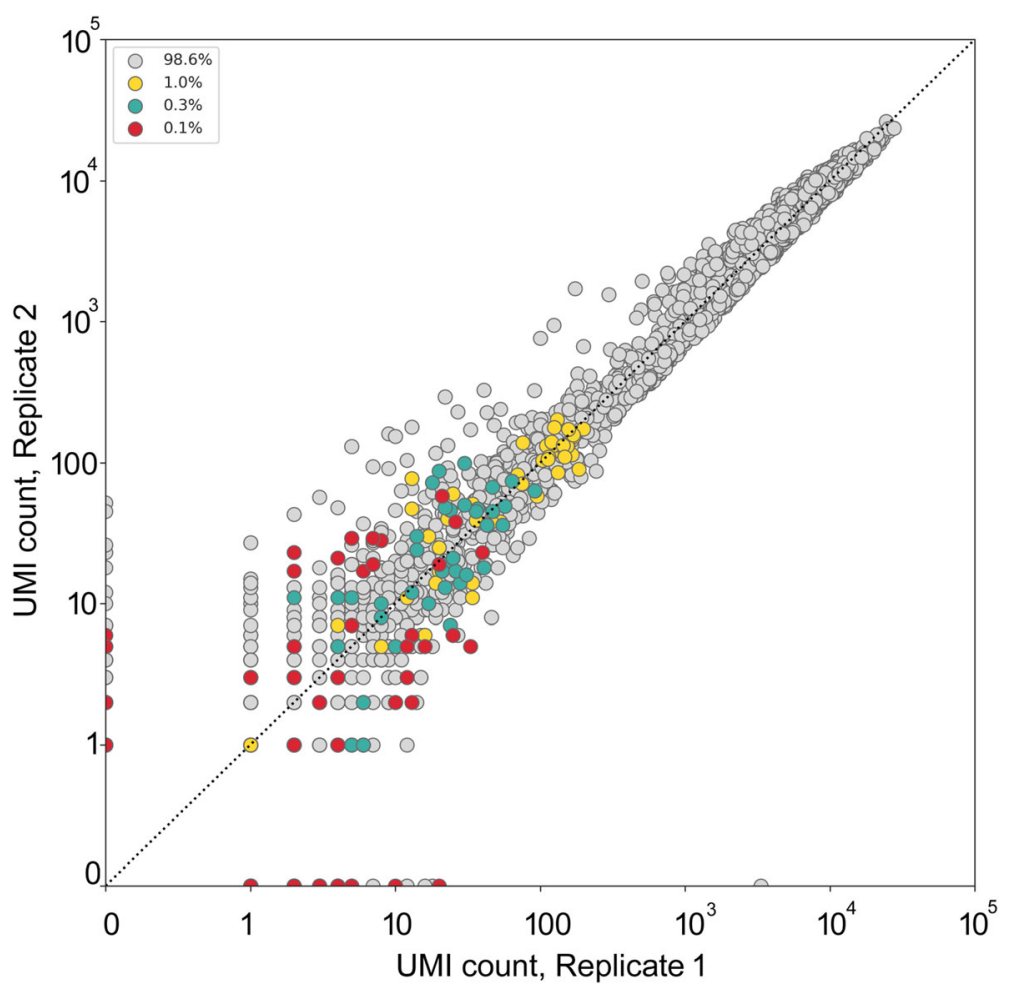

Fig. 4 Reproducibility between replicates. Grey dots - known fixed and polymorphic insertions of individual D1, yellow dots - individual specific insertions of D2 (1\%), green dots - individual specific insertions of D3 (0.3\%), red dots - individual specific insertions of D4 (0.1\%)

PCR with the initial template as described above. In all cases, we were unable to detect PCR products of expected length confirming that candidate Alu insertions from 5' flank libraries were artifacts.

To further proof the developed copy-capture approach we identified cancer-specific insertions of another family - L1HS in the same set of paired tumor/normal samples. As amplification and sequencing of 5 'flanking regions of L1 insertions is yet challenging we generated only the library of 3' flanks, using the same experimental approach as for Alu 3' flank libraries. Genomic DNA was digested with another pair of restriction enzymes and ligated to adapted with the same pan-handle like structure (see methods for details). After linear amplification with L1HS specific biotinylated primer 3' flank copies were extracted with streptavidin-coated magnetic beads and amplified in the subsequent 2 round PCR to generate ready-for-sequencing sample-barcoded libraries. Same as

Table 3 Candidate tumor-specific Alu insertions

\begin{tabular}{|c|c|c|c|c|c|c|c|}
\hline Sample & RE family & Chromosome & Position & UMI count in $5^{\prime}$ flank library & 3' flank Length & UMI count in $3^{\prime}$ flank library & PCR validation \\
\hline \multirow[t]{3}{*}{ CCC8 } & AluYa5 & 8 & $137,710,278$ & 4 & 44 & 0 & No \\
\hline & AluYa5 & 1 & $39,888,222$ & 2 & 544 & 0 & No \\
\hline & AluYb8 & 5 & $94,414,349$ & 2 & 218 & 0 & No \\
\hline \multirow[t]{2}{*}{ ccc9 } & AluYb8 & 5 & $77,457,780$ & 2 & 89 & $148(+397)^{a}$ & - \\
\hline & AluYb8 & 7 & $43,035,359$ & 3 & 137 & $2(+1)^{a}$ & $\mathrm{No}^{\mathrm{b}}$ \\
\hline \multirow[t]{3}{*}{ CCC11 } & AluYa5 & 8 & $80,855,361$ & 2 & 212 & $456(+283)^{a}$ & - \\
\hline & AluYa5 & 4 & $103,090,594$ & 2 & 20 & 0 & No \\
\hline & AluYa5 & 1 & $9,512,325$ & 2 & 108 & 0 & N/A \\
\hline $\mathrm{CCC} 13$ & AluYa5 & 16 & $70,789,164$ & 2 & 123 & 0 & No \\
\hline CCC14 & AluYa5 & 10 & $80,758,919$ & 2 & 423 & 0 & No \\
\hline
\end{tabular}

a also found in corresponding normal sample

b - PCR product detected in both tumor and normal samples

$\mathrm{N} / \mathrm{A}$ - impossible to design primers due to insertion into other repetitive sequences 
for Alu libraries, template DNA stays intact and is used for validation by locus-specific PCR. For each library we obtained a minimum of 219,970 reads and the difference between tumor and normal samples in each pair was less than 2-fold. In six paired samples combined we found 1119 L1HS insertions including 854 reference insertions (hg38), 188 previously known non-reference insertions (present in 1000 genomes, dbRIP or euL1db [27]) and 77 previously unknown non-reference insertions. We identified 10 tumor-specific candidate insertions in 5 out of 6 tumor/normal sample pairs (see Table 4). For 10 candidate insertions, we designed primers and performed locus-specific PCR with the initial template (see Methods). For 4 out of 10 insertions we obtained PCR product of expected length with tumor but not corresponding normal sample. For 3 other insertions we obtained PCR product in both tumor and normal samples and for the rest of insertions $(n=3)$ we failed to get PCR product. The obtained PCR products for 4 tumorspecific insertions were sequenced by Sanger method. In all cases we confirmed tumor-specific L1HS insertions.

\section{Discussion}

In the recent years it is becoming widely accepted that RE transcriptional and transpositional activity can play a significant role in cancer. New insertions can disrupt cellular genes (including those involved in malignant transformation) either by direct integration into a gene [28, 29] or via recombination-mediated chromosomal rearrangement [16]. A recent study by Cajuso et al. [30] showed that the amount of cancer-specific L1 insertions in colorectal cancer correlates with poor prognosis. With the growing capacity of modern sequencing machines, whole genome sequencing price is decreasing. However, the discovery of somatic variants including somatic insertions of RE in cancer cells still requires high sequencing coverage making analysis of hundreds of samples quite expensive. The identification of insertions present in a minor fraction $(1-10 \%)$ of tumor cells is not possible at all at the commonly used whole-genome sequencing depth. These insertions could be of particular interest as they can characterize a subclone that is functionally different from other tumor cells and can be a source of relapse in course of therapy. Thus, the development of cheap, sensitive, and simple methods for tumor-specific RE identification is desired. Here we present a method that combines high sensitivity, reliability and requires a moderate number of sequences per sample thus allowing the screening of hundreds of samples at a reasonable cost.

We use a modified selective amplification approach $[22,31]$ to generate the library of Alu flanks. As was shown previously [21] and in the current study selective amplification-based methods are very specific and have high (90\% and more) target sequence (flanks of young Alu or L1) content in the resulting library. This approach gives an approximate enrichment of 3000-fold in target RE flanking sequences compared to wholegenome shotgun libraries. The resulting library for individual D1 includes 2367 (2046 reference hg38 and 321 non-reference) or 3042 (2617 reference hg38 and 425 non-reference) previously known AluYa5 insertions depending on sequencing depth $(632,732$ and $35,488,107$ sequencing reads respectively). This number of insertions (3042) makes $91.5 \%$ of the average number of fullsize AluYa5 insertions (3324) detected in an individual genome by whole genome sequencing (evaluated from 1000 genomes [26, 32]).

The significant drawback of this approach is the inability to identify TSD - the hallmark of the retrotransposition event. As a result, even with very high sensitivity and vigorous artifact filtration pipelines, many potential insertions are not confirmed by the locus-specific PCR. This limitation can be overcome by the amplification of

Table 4 Candidate tumor-specific L1HS insertions

\begin{tabular}{|c|c|c|c|c|}
\hline Sample & Chromosome & Position & UMI count in 3' flank library & PCR validation \\
\hline \multirow[t]{2}{*}{$\overline{\mathrm{CCC} 8}$} & 8 & $137,421,701$ & 3 & $\mathrm{No}^{\mathrm{b}}$ \\
\hline & $x$ & $144,010,198^{a}$ & 4 & $\mathrm{No}^{\mathrm{b}}$ \\
\hline \multirow[t]{2}{*}{ CCC9 } & 5 & $28,280,060$ & 13 & Yes \\
\hline & 8 & $16,154,249$ & 3 & $\mathrm{No}^{\mathrm{b}}$ \\
\hline \multirow[t]{3}{*}{ CCC10 } & 20 & $53,163,810$ & 2 & No \\
\hline & 5 & $151,055,147$ & 2 & No \\
\hline & 8 & $128,834,433$ & 2 & No \\
\hline \multirow[t]{2}{*}{ CCC11 } & 3 & $138,985,467$ & 7 & Yes \\
\hline & 3 & $155,181,290$ & 16 & Yes \\
\hline $\mathrm{CCC} 13$ & $x$ & $38,440,949$ & 13 & Yes \\
\hline
\end{tabular}

a Possibly $3^{\prime}$ transduction

b - PCR product detected in both tumor and normal samples 
both flanking regions $\left(5^{\prime}\right.$ and $\left.3^{\prime}\right)$. However, this approach is not reliable when detecting insertions present in a minor fraction of cells if different aliquots of DNA from the same sample are used for library preparation and validation. Here we consecutively amplify both adjacent genomic regions of each insertion from the same template which significantly increases the chance to retrieve both flanks and identify TSD. In addition, sequence of one flank is used to validate the insertion found in the library of another flank: when we know the insertion coordinate from one library we can predict the length of another flank as we use restrictase for DNA fragmentation. If the corresponding sequence has suitable length for amplification and sequencing but not found in another flank library - this indicates false insertion. Moreover, template genomic DNA used for libraries preparation stays intact and can be used for locusspecific PCR to confirm insertions. Using spike-in controls we show that this approach can indeed produce reliable results. Most of the insertions that have corresponding sequences in $5^{\prime}$ and $3^{\prime}$ flank libraries were confirmed by locus-specific PCR from the same DNA template. We also directly characterized the sensitivity of the method. We show that the majority of insertions present in as little as in $1 \%$ of cells can be reliably (in two replicates) identified with the sequencing depth of only 2 million of $150+150$ paired reads (of $0.6 \mathrm{~Gb}$ ) per sample. This corresponds to $0.2 \mathrm{x}$ human genome coverage. It should be mentioned that uneven amplification efficiency of different flanking sequences results in different sequencing coverage for each particular RE insertion. As it was shown previously [21] amplification efficiency is proportional to flank length and also depends on other structural features of RE and its flanking region. This amplification and sequencing bias can be considered as a potential limitation of the proposed method as a portion of insertions is lost. However, such bias is characteristic for all PCR based methods. The proposed method is easily adapted to high-throughput formats and could be automated which gives a very powerful and cost-effective tool for researchers aiming to study hundreds of samples for new RE insertions. This method can be combined with the previously designed approach to advanced enrichment for rare somatic insertions [21]. Such combination could dramatically increase reliability and decrease the cost of somatic RE identification assay. The developed protocol for testing sensitivity of RE detection methods could help researchers in the field to evaluate and elevate the efficacy of existing and developing methods.

Unlike L1 insertions, tumor-specific Alu insertions are rare in cancers including colorectal cancer [33]. As a proof of concept, we searched for such events in 6 paired colorectal tumor/normal samples. With the sequencing depth sufficient for the identification of most insertions present in $1 \%$ of cells we detected 10 potentially tumor-specific AluYa5 insertions in the 5' flank library. These candidate insertions have 2-4 UMI indicating their presence in a minor percentage of tumor cells. None of these candidates were confirmed in the corresponding 3' flank library or were found in both tumor and normal sample $3^{\prime}$ libraries. Concordantly, none of these insertions were validated by locus-specific PCR. This finding indicates high reliability of the method: comparing sequences obtained for both flanks from the same template allows distinguishing true insertions from false candidates.

To further validate the method, we searched for tumor specific L1HS insertions in the same set of samples using the same methodological approach. We identified 10 candidate insertions with UMI counts ranging from 2 to 16 and fully confirmed 4 of them by orthogonal approach. These results indicate successful application of our method on real samples, and at the same time, demonstrate the necessity for sequencing of both flanks for reliable tumor-specific RE identification. Additionally, the identified tumor specific L1HS insertions indicate that at least 3 out of 6 analyzed colorectal tumor samples were subjected to retropositional activity. At the same time, we accurately showed absence of tumor specific Alu insertions in all 6 samples. Taken together, these facts indicate that Alu insertions in cancer occur much rarer than L1 and active L1 machinery alone is insufficient for Alu retropositional activity.

\section{Conclusion}

Most of the previously described techniques for targeted RE capture like RC-seq [34] use original DNA molecules which become unavailable after library preparation. Here we developed the new capture method that extracts copies of target molecules saving original DNA for downstream application such as validation assay, SNV sequencing or DNA methylation analysis. The described principle can be easily adapted for library enrichment by other target molecules after or instead of RE capture just by adding new target primers to the reaction. Thus, the proposed approach is characterized by high level of flexibility that can cover requirements of modern experimental demands for simultaneous analysis of different items or parameters in the same biological sample.

\section{Materials and methods}

\section{Sample collection and DNA isolation}

Six colorectal cancer samples were obtained during R0 partial colectomy from patients with stage II or III colorectal adenocarcinoma (5 patients) or carcinoma in situ (1 patient) treated at the A.N. Ryzhikh National Medical Research Centre for Coloproctology. The study was 
approved by the local ethical committee and all the patients gave standard informed consent. Tumor and normal tissue fragments were taken under the supervision of a pathomorphologist in the shortest possible time after intestinal resection (not more than $30 \mathrm{~min}$ ). DNA was extracted with QIAamp DNA Mini Kit (Qiagen) according to manufacturer's protocol.

PBMC of four healthy donors were isolated from peripheral blood by standard Ficoll-Paque (PanEco, Russia) centrifugation protocol. T-cells from different individuals were stained by the following antibodies: individual D2 CD3-eFluor450 (UCHT1, eBioscience), individual D3 - CD3-FITC (UCHT1, eBioscience), individual D4 CD3PC5 (UCHT1, Beckman Coulter). Cells of individual 1 were not stained. FACS sorting was performed in 5 replicates by BD FACS Aria III. Cells were gated based on side and forward scatter. Each cells mixture contained 50,000 cells of individual D1, 500 cells of individual D2, 150 cells of individual D3 and 50 cells of individual D4. After sorting three out of five aliquots were resorted to evaluate the resulting number of cells in the mixture. DNA from sorted cells was isolated using QIAGEN DNeasy Blood and Tissue Kit (Qiagen).

\section{Alu library preparation and sequencing}

To avoid possible contamination, we first prepared libraries from cell mixtures and sequenced them. After that we made libraries from DNA of individuals D1-D4 separately to identify individual-specific Alu insertions present in the genome of individuals D2, D3 or D4 and absent in the genome of individual D1.

Thirty ng of genomic DNA was digested by incubation in $10 \mu \mathrm{l}$ of $1 \mathrm{x}$ FD buffer with $5 \mathrm{U}$ of FspBI and $5 \mathrm{U}$ of Csp6I (all Thermo Fisher Scientific) for $30 \mathrm{~min}$ at $37^{\circ} \mathrm{C}$. For adapters ligation fragmented DNA was diluted by 20 $\mu \mathrm{l}$ of 1x FD Buffer with $20 \mu \mathrm{mol}$ of ATP (Thermo Fisher Scientific), $50 \mathrm{pmol}$ of SL-adapter (see Additional file 3 for oligo sequences), $10 \mathrm{U}$ of T4 DNA ligase (Thermo Fisher Scientific) and incubated at $22^{\circ} \mathrm{C}$ for $30 \mathrm{~min}$. Next, 50 pmol of antiSL-adapter, additional $5 \mathrm{U}$ of FspBI and Csp6I endonucleases were added and the mixture was incubated at $22^{\circ} \mathrm{C}$ for $30 \mathrm{~min}$ and $37^{\circ} \mathrm{C}$ for $30 \mathrm{~min}$. AntiSL-adapter inactivates SL-adapters and endonucleases decrease the number of ligation chimeric molecules. The reaction mixture was purified with $0.8 \mathrm{~V}$ of AmPure XP beads (Beckman Coulter) and eluted directly in $15 \mu \mathrm{l}$ of linear amplification reaction mixture containing $1 \mathrm{x}$ Encyclo Buffer, 1x Encyclo polymerase, $200 \mu \mathrm{M}$ of each dNTP (all Evrogen, Russia), $20 \mu \mathrm{M}$ of biotin-16-dCTP (Jena Bioscience, Germany), $0.2 \mu \mathrm{M}$ of 5 ' - flank oriented AluYa5 specific biotinylated primer (Additional file 3). The linear amplification profile was: $94^{\circ} \mathrm{C}$ for $3 \mathrm{~min}$, followed by 30 cycles of $20 \mathrm{~s}$ at $94^{\circ} \mathrm{C}, 20 \mathrm{~s}$ at $65^{\circ} \mathrm{C}$ and 1 min at $72{ }^{\circ} \mathrm{C}$ with ramp rate $1{ }^{\circ} \mathrm{C} / \mathrm{s}$. After amplification the product was mixed with $3 \mu \mathrm{l}$ of MyOne Streptavidin C1 Dynabeads (Thermo Fisher Scientific) resuspended in $1 \mathrm{x}$ Encyclo buffer and incubated for $15 \mathrm{~min}$ at room temperature with permanent rotation. Linear amplification product was eluted by $10 \mu \mathrm{l}$ of $\mathrm{mQ}$ water from streptavidin coated beads, purified by $1.5 \mathrm{~V}$ of AmPure $\mathrm{XP}$ beads and used for subsequent exponential PCRs. The supernatant was supplemented by $1 \mathrm{x}$ Encyclo polymerase, $20 \mu \mathrm{M}$ of biotin-16-dCTP and $0.2 \mu \mathrm{M}$ of AluYa5 specific 3' - flank oriented biotinylated primer and used for the 2nd linear amplification to obtain linear DNA fragments of opposite (3'-) Alu flanks. The amplification profile, capture and purification condition were the same as for $5^{\prime}$ - flank libraries. The remaining supernatant containing restricted and ligated template genomic DNA was used for the downstream validation of detected insertions by locus-specific PCR (see below). The captured linear amplicons (copies) were used in separate (for each flank) $25 \mu \mathrm{l} \mathrm{PCR} \mathrm{reactions} \mathrm{containing} 200 \mu \mathrm{M}$ of each of dNTP, $0.2 \mu \mathrm{M}$ of the Alu specific primer (see Additional file 3), adapter specific primer korNxtSt19ok and $1 \mathrm{x}$ Encyclo polymerase in 1x Encyclo Buffer. The amplification profile was: $2 \mathrm{~min}$ at $94^{\circ} \mathrm{C}$ followed by 10 cycles of $20 \mathrm{~s}$ at $94{ }^{\circ} \mathrm{C}, 20 \mathrm{~s}$ at $60^{\circ} \mathrm{C}, 1 \mathrm{~min}$ at $72^{\circ} \mathrm{C}$ with ramp rate $1{ }^{\circ} \mathrm{C} / \mathrm{s}$. One $\mu \mathrm{l}$ of the obtained PCR product was used in the second $25 \mu \mathrm{l} \mathrm{PCR}$ reaction containing $200 \mu \mathrm{M}$ of each dNTP, $0.2 \mu \mathrm{M}$ of each Nextera Indexing primers and 1x Encyclo polymerase in 1x Encyclo Buffer amplified for 12 cycles using the same amplification profile as for the first PCR. Second PCR products were purified with AmPure XP beads, mixed equimolarly and sequenced on Illumina NextSeq paired end $150+150$. Reference libraries from single individuals were sequenced separately on MiSeq paired end $150+150$.

DNA from colorectal cancer samples was processed exactly in the same way with two additional libraries containing AluYb8 flanks (see Additional file 3) prepared for each sample pair. Libraries were sequenced on Illumina NextSeq paired end $150+150$.

\section{L1HS library preparation and sequencing}

Thirty ng of genomic DNA was digested by incubation in $10 \mu \mathrm{l}$ of $1 \mathrm{x}$ FD buffer with $5 \mathrm{U}$ of FspBI and $5 \mathrm{U}$ of TaqI (all Thermo Fisher Scientific) for $30 \mathrm{~min}$ at $37^{\circ} \mathrm{C}$ and $30 \mathrm{~min}$ at $65^{\circ} \mathrm{C}$. For adapters ligation fragmented DNA was diluted by $20 \mu \mathrm{l}$ of $1 \mathrm{x}$ FD Buffer with $20 \mu \mathrm{mol}$ of ATP (Thermo Fisher Scientific), $50 \mathrm{pmol}$ of SLadapter-2 (see Additional file 3 for oligo sequences), 10 $\mathrm{U}$ of T4 DNA ligase (Thermo Fisher Scientific) and incubated at $22^{\circ} \mathrm{C}$ for $30 \mathrm{~min}$. Next, $50 \mathrm{pmol}$ of antiSLadapter-2, additional $5 \mathrm{U}$ of FspBI and TaqI endonucleases were added and the mixture was incubated at $22^{\circ} \mathrm{C}$ for $30 \mathrm{~min}$ and $37^{\circ} \mathrm{C}$ for $30 \mathrm{~min}$. The reaction mixture was purified with $0.8 \mathrm{~V}$ of AmPure XP beads (Beckman 
Coulter) and eluted directly in $15 \mu \mathrm{l}$ of linear amplification reaction mixture containing 1x Encyclo Buffer, 1x Encyclo polymerase, $200 \mu \mathrm{M}$ of each dNTP (all Evrogen, Russia), $20 \mu \mathrm{M}$ of biotin-16-dCTP (Jena Bioscience, Germany), $0.2 \mu \mathrm{M}$ of 3'- flank oriented L1HS specific biotinylated primer (Additional file 3). The linear amplification profile was: $94{ }^{\circ} \mathrm{C}$ for $3 \mathrm{~min}$, followed by 30 cycles of $20 \mathrm{~s}$ at $94{ }^{\circ} \mathrm{C}, 20 \mathrm{~s}$ at $55^{\circ} \mathrm{C}$ and $1 \mathrm{~min}$ at $72^{\circ} \mathrm{C}$ with ramp rate $1{ }^{\circ} \mathrm{C} / \mathrm{s}$. After amplification the product was mixed with $3 \mu \mathrm{l}$ of MyOne Streptavidin C1 Dynabeads (Thermo Fisher Scientific) resuspended in 1x Encyclo buffer and incubated for $15 \mathrm{~min}$ at room temperature with permanent rotation. Linear amplification product was eluted by $10 \mu \mathrm{l}$ of $\mathrm{mQ}$ water from streptavidin coated beads, purified by $1.5 \mathrm{~V}$ of AmPure XP beads and used for subsequent exponential PCRs as described for Alu using L1-specific primer (see Additional file 3) and 18 cycles PCR for indexing.

The supernatant was supplemented by $1 \mathrm{x}$ Encyclo polymerase, $20 \mu \mathrm{M}$ of biotin-16-dCTP and $0.2 \mu \mathrm{M}$ each of L1 multiplex primer set with locus specific primer (designed after 3' flank sequencing) and used for the 2nd amplification to obtain DNA fragments of opposite (5') L1HS flanks. The amplification profile, capture and purification condition were the same as for 3'- flank libraries. The captured amplicons (copies) were indexed at the same condition as $3^{\prime}$ flanks amplicons, purified with AmPure XP beads, mixed equimolarly and sequenced on Illumina MiSeq paired end $150+150$.

\section{Identification of individual-specific insertions}

Raw sequencing reads were processed as described previously [24]. As a result, we obtained a metatable containing a list of identified AluYa5 insertions in all four individuals with their genomic positions and read counts for each of the four individuals. Based on read counts for known fixed AluYa5 insertions we identified the value of first quartile (Q1) in each individual dataset. Then, using the metatable, we found polymorphic insertions that had a read count $>=\mathrm{Q} 1$ in one of the individuals D2, D3 or D4 dataset and 0 reads in all other individuals. To validate the insertions found by the computational pipeline, we searched for their flanking sequences in raw sequencing reads allowing for 1 mismatch.

\section{Validation of RE insertions by locus-specific PCR}

Primers for the specific genomic regions were designed with primer-blast and GeneRunner programs. Twentyfive $\mu \mathrm{l}$ PCR reactions containing $200 \mu \mathrm{M}$ of each of dNTP, $0.2 \mu \mathrm{M}$ of each of forward and reverse locusspecific primers (Additional file 3) and 1x Encyclo polymerase in 1x Encyclo Buffer. The amplification profile was as follows: $2 \mathrm{~min}$ at $94{ }^{\circ} \mathrm{C}$ followed by 35 cycles of $20 \mathrm{~s}$ at $94{ }^{\circ} \mathrm{C}, 20 \mathrm{~s}$ at $60^{\circ} \mathrm{C}, 1 \mathrm{~min}$ at $72^{\circ} \mathrm{C}$.

\section{Supplementary Information}

The online version contains supplementary material available at https://doi. org/10.1186/s13100-020-00228-6.

Additional file 1: Supplementary Table 1. A detailed description of individual-specific Alu insertions.

Additional file 2: Supplementary Table 2. TSD identification.

Additional file 3: Supplementary Table 3. Oligonucleotide sequences used for library preparation and locus-specific PCR.

\section{Acknowledgements}

We thank Alexey Kurnosov and Anna Miroshnichenkova for reading and comment the manuscript.

\section{Authors' contributions}

IZM and AYK initiated and designed the study. AYK designed

oligonucleotides and performed method sensitivity testing. SZU, GAN and AOS performed bioinformatic analysis. EAK performed fluorescent sorting. MVS performed retroelement insertion identification in cancer samples and validation assay. YAS, VPS, MYB, AST, AVS and AVK collected and

characterized the samples. IZM and AVS performed the sequencing. IZM,

AYK and YBL wrote the manuscript. All authors have read and approved the manuscript.

\section{Funding}

This work was supported by Russian Science Foundation grant 18-14-00244 to IZM.

\section{Availability of data and materials}

The sequence reads are available from the Sequence Read Archive (SRA) under the accession number PRJNA657558.

\section{Ethics approval and consent to participate}

The study was approved by the local ethics committee and conducted in accordance with the Declaration of Helsinki.

\section{Consent for publication}

Not applicable.

\section{Competing interests}

The authors declare that they have no competing interests.

\section{Author details}

${ }^{1}$ Shemyakin-Ovchinnikov Institute of Bioorganic Chemistry, Moscow, Russia. ${ }^{2}$ Dmitry Rogachev National Medical and Research Center of Pediatric Hematology, Oncology and Immunology, Moscow, Russia. ${ }^{3}$ Ryzhikh National Medical Research Centre for Coloproctology of the Ministry of Health of Russia, Moscow, Russia. ${ }^{4}$ V.I. Kulakov National Medical Research Center for Obstetrics, Gynecology and Perinatology, Moscow, Russia. ${ }^{5}$ Engelhardt Institute of Molecular Biology, Russian Academy of Sciences, Moscow, Russia. ${ }^{6}$ Central European Institute of Technology, Masaryk University, Brno, Czech Republic.

Received: 16 September 2020 Accepted: 1 December 2020 Published online: 14 December 2020

References

1. Penzkofer $T$, Jäger $M$, Figlerowicz $M$, Badge $R$, Mundlos $S$, Robinson PN, et al. L1Base 2: more retrotransposition-active LINE-1s, more mammalian genomes. Nucleic Acids Res. 2017;45:D68-73.

2. Feusier J, Watkins WS, Thomas J, Farrell A, Witherspoon DJ, Baird L, et al. Pedigree-based estimation of human mobile element retrotransposition rates. Genome Res. 2019;29:1567-77.

3. Kazazian HH, Moran JV. Mobile DNA in health and disease. N Engl J Med. 2017;377:361-70.

4. Burns KH. Transposable elements in cancer. Nat Rev Cancer. 2017;17:415-24.

5. Helman E, Lawrence MS, Stewart C, Sougnez C, Getz G, Meyerson M. Somatic retrotransposition in human cancer revealed by whole-genome and exome sequencing. Genome Res. 2014;24:1053-63. 
6. Tubio JMC, Li Y, Ju YS, Martincorena I, Cooke SL, Tojo M, et al. Mobile DNA in cancer. Extensive transduction of nonrepetitive DNA mediated by L1 retrotransposition in cancer genomes. Science. 2014;345:1251343.

7. Schauer SN, Carreira PE, Shukla R, Gerhardt DJ, Gerdes P, Sanchez-Luque FJ, et al. L1 retrotransposition is a common feature of mammalian hepatocarcinogenesis. Genome Res. 2018:28:639-53.

8. Coufal NG, Garcia-Perez JL, Peng GE, Yeo GW, Mu Y, Lovci MT, et al. L1 retrotransposition in human neural progenitor cells. Nature. 2009;460: 1127-31.

9. Evrony GD, Cai X, Lee E, Hills LB, Elhosary PC, Lehmann HS, et al. Singleneuron sequencing analysis of $\mathrm{L} 1$ retrotransposition and somatic mutation in the human brain. Cell. 2012;151:483-96.

10. Kano H, Godoy I, Courtney C, Vetter MR, Gerton GL, Ostertag EM, et al. L1 retrotransposition occurs mainly in embryogenesis and creates somatic mosaicism. Genes Dev. 2009;23:1303-12.

11. van den Hurk JAJM, Meij IC, del Carmen Seleme M, Kano H, Nikopoulos K, Hoefsloot LH, et al. L1 retrotransposition can occur early in human embryonic development. Hum Mol Genet. 2007;16:1587-92.

12. Erwin JA, Marchetto MC, Gage FH. Mobile DNA elements in the generation of diversity and complexity in the brain. Nat Rev Neurosci. 2014;15:497-506.

13. Kurnosov AA, Ustyugova SV, Nazarov VI, Minervina AA, Komkov AY, Shugay $M$, et al. The evidence for increased L1 activity in the site of human adult brain neurogenesis. PLoS One. 2015;10:e0117854.

14. Upton KR, Gerhardt DJ, Jesuadian JS, Richardson SR, Sánchez-Luque FJ, Bodea GO, et al. Ubiquitous L1 mosaicism in hippocampal neurons. Cell. 2015;161:228-39.

15. Goubert C, Thomas J, Payer LM, Kidd JM, Feusier J, Watkins WS, et al. TypeTE: a tool to genotype mobile element insertions from whole genome resequencing data. Nucleic Acids Res. 2020;48:e36.

16. Rodriguez-Martin B, Alvarez EG, Baez-Ortega A, Zamora J, Supek F, Demeulemeester J, et al. Pan-cancer analysis of whole genomes identifies driver rearrangements promoted by LINE-1 retrotransposition. Nat Genet. 2020;52:306-19.

17. Loh JW, Ha H, Lin T, Sun N, Burns KH, Xing J. Integrated Mobile element scanning (ME-scan) method for identifying multiple types of polymorphic mobile element insertions. Mob DNA. 2020;11:12.

18. Tang Z, Steranka JP, Ma S, Grivainis M, Rodić N, Huang CRL, et al. Human transposon insertion profiling: analysis, visualization and identification of somatic LINE-1 insertions in ovarian cancer. Proc Natl Acad Sci U S A. 2017; 114:E733-40.

19. Ewing AD, Gacita A, Wood LD, Ma F, Xing D, Kim M-S, et al. Widespread somatic $\mathrm{L} 1$ retrotransposition occurs early during gastrointestinal cancer evolution. Genome Res. 2015;25:1536-45.

20. Kvikstad EM, Piazza P, Taylor JC, Lunter G. A high throughput screen for active human transposable elements. BMC Genomics. 2018;19:115.

21. Komkov AY, Minervina AA, Nugmanov GA, Saliutina MV, Khodosevich KV, Lebedev YB, et al. An advanced enrichment method for rare somatic retroelement insertions sequencing. Mob DNA. 2018;9:31.

22. Mamedov IZ, Arzumanyan ES, Amosova AL, Lebedev YB, Sverdlov ED. Whole-genome experimental identification of insertion/deletion polymorphisms of interspersed repeats by a new general approach. Nucleic Acids Res. 2005;33:e16

23. Evrony GD, Lee E, Park PJ, Walsh CA. Resolving rates of mutation in the brain using single-neuron genomics. Elife. 2016;5.

24. Nugmanov GA, Komkov AY, Saliutina MV, Minervina AA, Lebedev YB, Mamedov IZ. A pipeline for the error-free identification of somatic Alu insertions in high-throughput sequencing data. Mol Biol (Mosk). 2019:53: $154-65$

25. Kivioja T, Vähärautio A, Karlsson K, Bonke M, Enge M, Linnarsson S, et al. Counting absolute numbers of molecules using unique molecular identifiers. Nat Methods. 2011;9:72-4.

26. Sudmant PH, Rausch T, Gardner EJ, Handsaker RE, Abyzov A, Huddleston J, et al. An integrated map of structural variation in 2,504 human genomes. Nature. 2015;526:75-81.

27. Mir AA, Philippe C, Cristofari G. euL1db: the European database of L1HS retrotransposon insertions in humans. Nucleic Acids Res. 2015;43:D43-7.

28. Pitkänen E, Cajuso T, Katainen R, Kaasinen E, Välimäki N, Palin K, et al. Frequent L1 retrotranspositions originating from TTC28 in colorectal cancer. Oncotarget. 2014;5:853-9.
29. Shukla R, Upton KR, Muñoz-Lopez M, Gerhardt DJ, Fisher ME, Nguyen T, et al. Endogenous retrotransposition activates oncogenic pathways in hepatocellular carcinoma. Cell. 2013;153:101-11.

30. Cajuso T, Sulo P, Tanskanen T, Katainen R, Taira A, Hänninen UA, et al. Retrotransposon insertions can initiate colorectal cancer and are associated with poor survival. Nat Commun. 2019;10:4022.

31. Mamedov I, Batrak A, Buzdin A, Arzumanyan E, Lebedev Y, Sverdlov ED. Genome-wide comparison of differences in the integration sites of interspersed repeats between closely related genomes. Nucleic Acids Res. 2002;30:e71.

32. Stewart C, Kural D, Strömberg MP, Walker JA, Konkel MK, Stütz AM, et al. A comprehensive map of mobile element insertion polymorphisms in humans. PLoS Genet. 2011;7:e1002236.

33. Lee E, Iskow R, Yang L, Gokcumen O, Haseley P, Luquette LI, et al. Landscape of somatic retrotransposition in human cancers. Science. 2012. 337:967-71.

34. Baillie JK, Barnett MW, Upton KR, Gerhardt DJ, Richmond TA, De Sapio F, et al. Somatic retrotransposition alters the genetic landscape of the human brain. Nature. 2011;479:534-7.

\section{Publisher's Note}

Springer Nature remains neutral with regard to jurisdictional claims in published maps and institutional affiliations.

\section{Ready to submit your research? Choose BMC and benefit from:}

- fast, convenient online submission

- thorough peer review by experienced researchers in your field

- rapid publication on acceptance

- support for research data, including large and complex data types

- gold Open Access which fosters wider collaboration and increased citations

- maximum visibility for your research: over $100 \mathrm{M}$ website views per year

At BMC, research is always in progress.

Learn more biomedcentral.com/submissions 